\title{
OBSERVAÇÃO DE PARÂMETROS CARDIOVASCULARES EM PACIENTES INFARTADOS FRENTE A VISITA DE FAMILIARES
}

\author{
Edna Ikumi Umebayashi Takahashi *
}

TAKAHASHI, E. I. U. Observação de parâmetros cardiovasculares em pacientes infartados frente a visita de familiares. Rev. Esc. Enf. USP, São Paulo, 14(1):9-19, 1980.

Trata-se de um estudo feito com vinte pacientes infartados, nos quais examinou-se a pressão arterial e freqüencia cardíaca quando de um evento social - a visita de familiares. Pelos resultados obtidos verificou-se que os pacientes infartados apresentaram alterações emocionais nessa ocasião.

\section{Da definição do problema à realização do trabalho}

Durante a nossa experiência em uma Unidade de Tratamento Intensivo (UTI), especializada no atendimento de pacientes infartados, observamos que uma grande maioria desses, apresentava reações emocionais quando da visita de familiares. Em alguns pacientes foi possivel ainda observar, no monitor central, episódios de arritmias durante esse evento social.

Sabemos que as emoções e conseqüente descarga de catecolaminas aumentam o trabalho cardíaco. Para o paciente com Infarto do Miocárdio (IM) as respostas cardiovasculares a uma emoção podem ser precipitadoras de sérias complicações.

$\mathrm{Na}$ tentativa de aferirmos objetivamente essas eventuais alterações decidimos iazer um estudo de observação dos parâmetros cardiovasculares quando do estímulo emocional externo que seria representado pela visita de familiares.

Baseando-nos na afirmação de SECOR ${ }^{3}$, de que "a tensão emocional, tanto quanto a atividade física, aumenta o trabalho cardíaco" e na de $\operatorname{LEE}^{2}$, de que "é natural o uso dos métodos de esfigmomanometria e ausculta cardíaca no estudo da resposta cardiovascular a um estresse emocional", resolvemos avaliar clinicamente, por meio da verificação da pressão arterial (PA) e freqüência cardíaca (FC), a influência da visita de familiares na evolução do doente infartado.

Decidimos realizar esse estudo clínico com os pacientes infartados, internados na UTI de um hospital governamental.

Foram escolhidos os pacientes com diagnóstico de IM, com prescrição de dieta leve, que estivessem conscientes na ocasião e não apresentassem temperatura oral superior a $37,8^{\circ} \mathrm{C}$.

* Professor Colaborador da disciplina Enfermagem Médico-Cirúrgica I da EEUSP. 
Após seleção do paciente, eram anotados, em ficha elaborada para esse fim (Anexo 1), os dados de identificação contidos na papeleta do paciente. Caso não fosse possivel completar os dados de identificação somente com a papeleta, estes eram colhidos posteriormente, durante a entrevista, pela enfermeira da Unidade.

A rotina de visitas na unidade permitia a entrada de visitante a partir de quatorze horas, podendo este permanecer no máximo até dezesseis horas. A primeira mensuração foi, pois, feita às treze horas, e o valor obtido nesta mensuração foi considerado como o basal do paciente, uma vez que o mesmo se encontrava em período de repouso.

As verificações da PA foram feitas por duas vezes em cada controle, com intervalo de dois minutos para obtenção de um dado mais preciso, considerando-se, para fins de tratamento estatístico, a média desses valores. A mensuração da FC foi feita no intervalo entre as duas verificações da PA. Para mensuração desses parâmetros empregamos as técnicas adotadas nessa Unidade abaixo descritas.

\section{Técnica de verificação da freqüência cardíaca}

Paciente em decúbito dorsal horizontal; a enfermeira escolhe, com auxílio do estetoscópio, na região precordial, um dos seguintes focos de ausculta:

\section{- foco mitral, na região apexiana;}

- foco tricúspide, na base do apêndice xifóide;

- foco aórtico, no segundo espaço intercostal direito, junto ao esterno; e

- foco pulmonar, no segundo espaço intercostal esquerdo, próximo ao bordo esternal.

A escolha do foco foi feita de acordo com cada paciente, ou seja, onde melhor se transmitiam os ruídos. Encontrando o foco, a enfermeira fixava o estetoscópio no local com a mão direita e, com o auxílio de um relógio no braço esquerdo, contava os batimentos cardíacos auscultados durante um minuto. Caso houvesse qualquer dúvida, repetia a operação.

Técnica de verificação da pressão arterial.

A pressão arterial foi verificada de preferência no braço esquerdo, com o paciente em decúbito dorsal horizontal. Prendíamos o "cuff" no terço médio e superior do braço, tendo o cuidado de manter o mostrador do manômetro voltado para frente.

it.- 0 estetoscópio foi colocado na parte interna do terço inferior do braço. Insuflamos o "cuff" até a pressão de $200 \mathrm{mmHg}$ ou até que o pulso não fosse mais audível. $\mathrm{O}$ ar foi, então, gradualmente liberado e, com o aumento do fluxo sangüineo na artéria sob o "cuff", apareceram ruídos intercalados dando o ritmo dos batimentos cardíacos.

Consideramos, como pressão sistólica, o primeiro som obtido com a desinflação do "cuff" e, como pressão diastólica, o último som audivel. Além desses dois pontos, foram observadas as características dos sons, que sofriam mudanças gradativas pela redução da pressão no esfigmomanômetro. 
No momento que o visitante se apresentava na porta de entrada da Unidade, era feita a sua orientação quanto à visita, ressaltando-se principalmente a importância de manter conversação descontraída com o paciente, evitando trazer-lhe preocupações de casa. Nessa ocasião, procuramos conhecer o grau de parentesco do visitante.

Feita a orientação, o visitante era encaminhado até a unidade do paciente, quando registrávamos o horário de entrada do mesmo. Enquanto ocorria a interação paciente-visitante, observações eram feitas à distância, quanto às reações emotivas apresentadas por ambos e, ainda, quanto às alterações de ritmo cardíaco do paciente registradas no monitor central.

A segunda mensuração era feita imediatamente após a saida do visitante, registrando-se na ficha (Anexo 1) os valores encontrados da PA e da FC, como também o horário de saída e o tempo de permanência desse visitante.

No período de um ano e meio, conseguimos, com o auxílio dos enfermeiros dessa unidade, coletar dados de vinte pacientes (Anexo 2). Apesar da alta rotatividade de pacientes nesta Unidade, só conseguimos um pequeno número de doentes em nossa população, por duas razões básicas: houve alterações de rotina de visita e adoção de uma nova técnica para obtenção de parâmetros hemodinâmicos, que exigia a manipulação do doente infartado durante seu periodo de repouso, periodo este que correspondia ao horário de nossa primeira mensuração.

As respostas obtidas dos vinte pacientes serão apresentadas a seguir, com os devidos comentários.

\section{Dos resultados obtidos à análise dos dados}

Nas tabelas 1, 2 e 3 que seguem, apresentamos respectivamente os valores da freqüência cardíaca (FC), pressão arterial sistólica (PAS) e pressão arterial diastólica (PAD), obtidos nos pacientes infartados antes e após a visita de familiares, como também as diferenças entre os dois valores (antes-depois) de cada parâmetro.

TABELA 1

Idade, sexo e estado civil de pacientes infartados, valores da frequência cardíaca obtidos antes e após a visita de familiares e diferença entre as mesmas. São Paulo, 1976

\begin{tabular}{|c|c|c|c|c|c|c|}
\hline $\mathrm{N} .^{\circ}$ & Idade & Sexo & Estado Civil & Antes & Depois & $d(A-D)$ \\
\hline $\begin{array}{c}1 . \\
2 . \\
3 . \\
4 . \\
5 . \\
6 . \\
7 . \\
8 . \\
9 . \\
10 . \\
11 . \\
12 . \\
13 . \\
14 . \\
15 . \\
16 . \\
17 . \\
18 . \\
19 . \\
20 .\end{array}$ & $\begin{array}{l}61 \\
66 \\
47 \\
63 \\
65 \\
60 \\
74 \\
61 \\
47 \\
49 \\
46 \\
57 \\
45 \\
57 \\
45 \\
60 \\
62 \\
50 \\
70 \\
61\end{array}$ & $\begin{array}{l}\mathbf{M} \\
\mathbf{M} \\
\mathbf{M} \\
\mathbf{M} \\
\mathbf{M} \\
\mathbf{M} \\
\mathbf{M} \\
\mathbf{M} \\
\mathbf{M} \\
\mathbf{F} \\
\mathbf{M} \\
\mathbf{M} \\
\mathbf{M} \\
\mathbf{M} \\
\mathbf{M} \\
\mathbf{M} \\
\mathbf{M} \\
\mathbf{M} \\
\mathbf{F} \\
\mathbf{M}\end{array}$ & $\begin{array}{l}\mathbf{S} \\
\mathbf{C} \\
\mathbf{C} \\
\mathbf{S} \\
\mathbf{C} \\
\mathbf{C} \\
\mathbf{C} \\
\mathbf{C} \\
\mathbf{C} \\
\mathbf{C} \\
\mathbf{C} \\
\mathbf{C} \\
\mathbf{C} \\
\mathbf{C} \\
\mathbf{C} \\
\mathbf{C} \\
\mathbf{C} \\
\mathbf{C} \\
\mathbf{C} \\
\mathbf{C}\end{array}$ & $\begin{array}{r}68 \\
90 \\
86 \\
58 \\
78 \\
92 \\
106 \\
82 \\
78 \\
82 \\
45 \\
124 \\
74 \\
68 \\
74 \\
80 \\
70 \\
90 \\
104 \\
71\end{array}$ & $\begin{array}{r}74 \\
90 \\
98 \\
74 \\
82 \\
100 \\
114 \\
92 \\
78 \\
82 \\
90 \\
138 \\
78 \\
98 \\
80 \\
80 \\
90 \\
126 \\
110 \\
71\end{array}$ & $\begin{array}{r}-6 \\
0 \\
-12 \\
-16 \\
-4 \\
-8 \\
-8 \\
-10 \\
0 \\
0 \\
-45 \\
-14 \\
-4 \\
-30 \\
-6 \\
0 \\
-10 \\
-36 \\
-6 \\
0\end{array}$ \\
\hline
\end{tabular}

$\mathbf{M}=$ masculino; $\mathbf{F}=$ feminino; $\mathbf{C}=$ casado; $\mathbf{S}=$ solteiro; $\mathbf{d}(\mathbf{A}-\mathrm{D})=$ Para cálculo da diferença 0 valor obtido antes da visita fol subtrádo, do valor obtido após a visita. 
Na Tabela 1, observamos que em quinze pacientes $(75 \%)$ o valor obtido antes da visita foi inferior ao obtido após a visita significando que, para esses, a FC após a visita encontrava-se elevada (de 4 a 45 unidades ou batimentos/minuto a mais). Em cinco pacientes $(25 \%)$ a d (A-D) foi nula, isto é, não houve alteração da FC após a visita de familiares.

TABELA 2

Idade, sexo e estado civil de pacientes infartados, valores da pressão arterial sistólica obtidos antes e e após a visita de familiares e diferença entre as mesmas. São Paulo, 1976

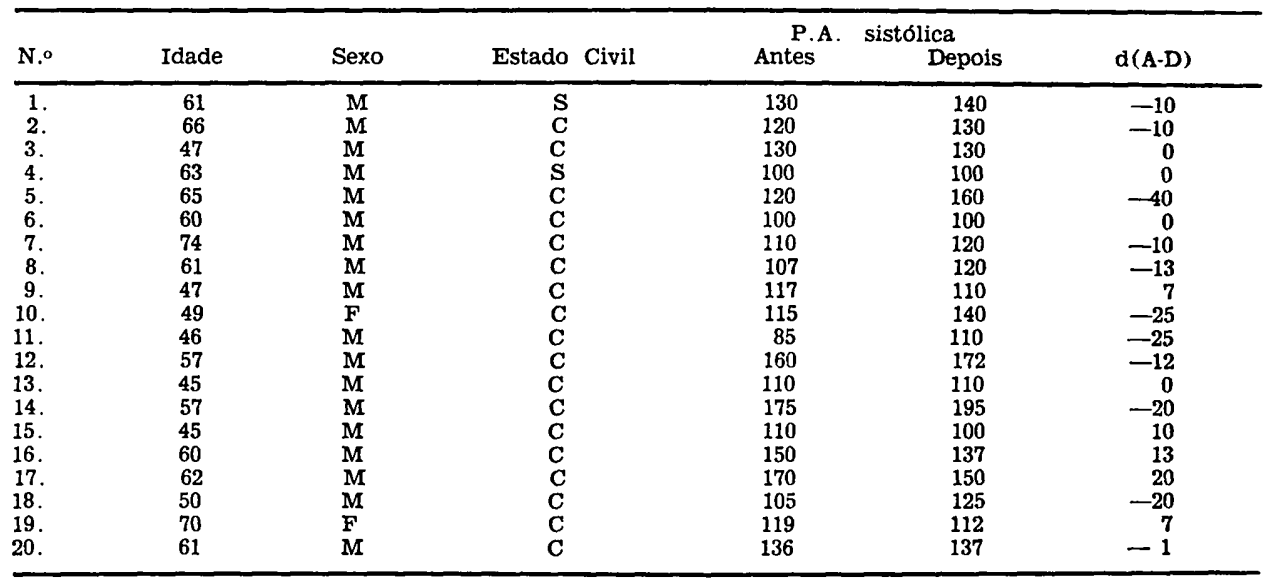

$\mathbf{M}=$ masculino; $\mathbf{F}=$ feminino; $\mathbf{C}=$ casado; $\mathbf{S}=$ solteiro; $\mathrm{d}(\mathrm{A} \cdot \mathrm{D})=$ Para cálculo da diferença $\mathrm{o}$ valor obtido antes da visita foi subtraído, do valor obtido após a visita.

Para a variável PAS, verificamos, pela Tabela 2, que em onze pacientes (55\%) esse parâmetro aumentou na segunda mensuração $(10$ a $40 \mathrm{mmHg})$, em cinco, $(25 \%$ ) diminuiu (de 7 a $20 \mathrm{mmHg}$ ) e em quatro (20\%) não houve alteração.

TABELA 3

Idade, sexo e estado civil de pacientes infartados, valores da pressão arterial diastólica obtidos antes e e após a visita de familiares e diferença entre as mesmas. São Paulo, 1976

\begin{tabular}{|c|c|c|c|c|c|c|}
\hline N.o & Idade & Sexo & Estado Civil & $\begin{array}{c}\text { P.A. } \\
\text { Antes }\end{array}$ & $\begin{array}{l}\text { lica } \\
\text { Depois }\end{array}$ & $\mathbf{d}(\mathbf{A}-\mathrm{D})$ \\
\hline $\begin{array}{l}1 . \\
2 . \\
3 . \\
4 . \\
5 . \\
6 . \\
7 . \\
8 . \\
9 . \\
10 . \\
11 . \\
12 . \\
13 . \\
14 . \\
15 . \\
16 . \\
17 . \\
18 . \\
19 . \\
20 .\end{array}$ & $\begin{array}{l}61 \\
66 \\
47 \\
63 \\
65 \\
60 \\
74 \\
61 \\
47 \\
49 \\
46 \\
57 \\
45 \\
57 \\
45 \\
60 \\
62 \\
50 \\
70 \\
61\end{array}$ & $\begin{array}{l}\mathbf{M} \\
\mathbf{M} \\
\mathbf{M} \\
\mathbf{M} \\
\mathbf{M} \\
\mathbf{M} \\
\mathbf{M} \\
\mathbf{M} \\
\mathbf{M} \\
\mathbf{F} \\
\mathbf{M} \\
\mathbf{M} \\
\mathbf{M} \\
\mathbf{M} \\
\mathbf{M} \\
\mathbf{M} \\
\mathbf{M} \\
\mathbf{M} \\
\mathbf{F} \\
\mathbf{M}\end{array}$ & $\begin{array}{l}\text { S } \\
\mathbf{C} \\
\mathbf{C} \\
\mathbf{S} \\
\mathbf{C} \\
\mathbf{C} \\
\mathbf{C} \\
\mathbf{C} \\
\mathbf{C} \\
\mathbf{C} \\
\mathbf{C} \\
\mathbf{C} \\
\mathbf{C} \\
\mathbf{C} \\
\mathbf{C} \\
\mathbf{C} \\
\mathbf{C} \\
\mathbf{C} \\
\mathbf{C} \\
\mathrm{C}\end{array}$ & $\begin{array}{r}70 \\
70 \\
90 \\
40 \\
80 \\
70 \\
80 \\
70 \\
70 \\
80 \\
66 \\
110 \\
60 \\
125 \\
80 \\
100 \\
110 \\
75 \\
78 \\
99\end{array}$ & $\begin{array}{r}90 \\
70 \\
90 \\
40 \\
90 \\
70 \\
85 \\
80 \\
80 \\
95 \\
80 \\
110 \\
60 \\
137 \\
70 \\
100 \\
90 \\
92 \\
85 \\
100\end{array}$ & $\begin{array}{r}-20 \\
0 \\
0 \\
0 \\
-10 \\
0 \\
-5 \\
-10 \\
-10 \\
-15 \\
-14 \\
0 \\
0 \\
-12 \\
10 \\
0 \\
20 \\
-17 \\
-7 \\
-1\end{array}$ \\
\hline
\end{tabular}

$\mathbf{M}=$ masculino; $\mathbf{F}=$ feminino; $\mathbf{C}=$ casado; $\mathbf{S}=$ solteiro; $\mathrm{d}(\mathrm{A}-\mathrm{D})=$ Para cálculo da diferença $\mathbf{0}$ valor obtido antes da visita foi subtraído, do valor obtido após a visita. 
Na Tabela 3 , referente a PAD, obtivemos em onze pacientes $(55 \%)$ valores maiores após a visita de familiares (de 1 a $20 \mathrm{mmHg}$ ); em dois $(2 \%)$, valores menores (10 e $20 \mathrm{mmHg}$ ) e em sete pacientes $(35 \%)$ não houve variações.

Por essas três tabelas iniciais observamos, portanto, que um número maior de pacientes apresentou valores dos três parâmetros aumentados depois da visita de familiares. A fim de verificar se essas variáveis em estudo (FC, PÁS e PAD) apresentavam alterações significativas antes e depois da visita de familiares, procedemos ao teste de " $t$ " para observações correlacionadas, uma vez que ambas as medidas foram realizadas no mesmo individuo. Para tanto, calculamos a média das diferenças de cada variável, as estimativas de suas respectivas variâncias e o valor do " $t$ " observado (t obs) (Tabela 4).

TABELA 4

Média das diferenças das variáveis em estudo, estimativa das variâncias e valores da estatística "t" observado. São Paulo, 1976

\begin{tabular}{l|ccc}
\hline \multicolumn{1}{c|}{ Variável } & $\begin{array}{c}\text { Média das } \\
\text { diferenças }\end{array}$ & $\begin{array}{c}\text { Estimativa da } \\
\text { variância }\end{array}$ & t obs. \\
\hline Frequiência cardíaca & $-10,75$ & 156,51 & $-3,84$ \\
Pressão arterial sistólica & $-6,45$ & 221,00 & $-1,94$ \\
Pressão arterial diastólica & $-4,55$ & 90,26 & $-2,14$ \\
\hline
\end{tabular}

Tendo comparado o "t obs" ao valor crítico de 0,05 percentil da distribuição " $t$ ", que equivale a $-1,729$, verificamos que, para as três variáveis, houve uma alteração significativa.

A seguir analisamos as diferenças entre os valores obtidos dos três parâmetros antes e após a visita de familiares, com algumas variáveis como idade, dia de internação, grau de parentesco e tempo de permanência.

TABELA 5

Distribuição dos pacientes infartados, internados em UTI, segundo a idade e a d(A-D) da frequiência cardíaca. São Paulo, 1976

\begin{tabular}{|c|c|c|c|c|c|c|c|}
\hline $\int_{\text {Idade }}^{\substack{d(A \cdot D) \\
\text { da } F C}}$ & zero & $0-10$ & $10-20$ & $20-30$ & $30-140$ & $40-150$ & Total \\
\hline $\begin{array}{l}40-160 \\
60-180\end{array}$ & $\begin{array}{l}2 \\
3\end{array}$ & $\begin{array}{l}2 \\
7\end{array}$ & $\begin{array}{l}2 \\
1\end{array}$ & 1 & 1 & 1 & $\begin{array}{r}9 \\
11\end{array}$ \\
\hline Total & 5 & 9 & 3 & 1 & 1 & 1 & 20 \\
\hline
\end{tabular}

TABELA 6

Distribuicão dos pacientes infartados, internados em UTI, segundo a idade e a d(A.D) da pressão arterial sistólica. São Paulo, 1976

\begin{tabular}{|c|c|c|c|c|c|c|c|c|}
\hline \multirow{2}{*}{$\underbrace{d(A-D) d a}_{\text {Idade }}$} & \multicolumn{2}{|c|}{$\begin{array}{l}\text { diferença } \\
\text { positiva }\end{array}$} & \multicolumn{2}{|c|}{ diferença } & \multicolumn{2}{|c|}{ negativa } & \multirow{2}{*}{$\begin{array}{l}\text { diferença } \\
\text { nula }\end{array}$} & \multirow{2}{*}{ Total } \\
\hline & $0-10$ & $10-20$ & $0-10$ & $10-\mid 20$ & $20-\mid 30$ & $30-\mid 40$ & & \\
\hline $\begin{array}{l}40-60 \\
60-180\end{array}$ & $\begin{array}{l}2 \\
1\end{array}$ & $\overline{2}$ & $\overline{4}$ & $\begin{array}{l}3 \\
1\end{array}$ & -2 & $\overline{1}$ & $\begin{array}{l}2 \\
2\end{array}$ & $\begin{array}{r}9 \\
11\end{array}$ \\
\hline Total & 3 & 2 & 4 & 4 & 2 & 1 & 4 & 20 \\
\hline
\end{tabular}


TABELA 7

Distribuição dos pacientes infartados, internados em UTI, segundo a idade e a d(A-D) da pressão arterial diastólica. São Paulo, 1976

\begin{tabular}{|c|c|c|c|c|c|c|}
\hline$\underbrace{d(A-D)}_{\text {Idade }} d a$ & ${ }_{0-10}^{d j}$ & $\begin{array}{l}\text { aça } \\
\text { va } \\
10-\mid 20\end{array}$ & $\begin{array}{r}d \\
n \\
0-10\end{array}$ & $\begin{array}{l}\text { nça } \\
\text { iva } \\
10-120\end{array}$ & $\begin{array}{c}\text { diferença } \\
\text { nula }\end{array}$ & Total \\
\hline $\begin{array}{l}40-60 \\
60-\mid 80\end{array}$ & 1 & $\overline{1}$ & $\begin{array}{l}1 \\
5\end{array}$ & $\begin{array}{l}4 \\
1\end{array}$ & $\begin{array}{l}3 \\
4\end{array}$ & $\begin{array}{r}9 \\
11\end{array}$ \\
\hline Total & 1 & 1 & 6 & 5 & 7 & 20 \\
\hline
\end{tabular}

Por essas tabelas constatamos que os pacientes da faixa etária de quarenta a sessenta anos tiveram alterações mais significativas dos três parâmetros, principalmente da FC, após a visita de familiares. Isto nos levou a crer que as expectativas com relação ao futuro dos indivíduos dessa faixa etária, podem ser fatores desencadeantes de ansiedade promovendo maior emotividade ao paciente na visita de familiares.

\section{TABELA 8}

Distribuição dos pacientes infartados, internados em UTI, segunđo o dia de internação e a d(A.D) da frequeência cardíaca. São Paulo, 1976

\begin{tabular}{|c|c|c|c|c|c|c|c|}
\hline $\begin{array}{l}\text { d(A.D) } \\
\text { Dia de da FC } \\
\text { internação }\end{array}$ & zero & $0-10$ & $10-\mid 20$ & $20-30$ & $30-140$ & $40-150$ & Total \\
\hline $\begin{array}{l}1.0^{\circ} \\
2 .{ }^{\circ} \\
3 . .^{\circ}\end{array}$ & $\begin{array}{l}1 \\
3 \\
1\end{array}$ & $\begin{array}{l}5 \\
3 \\
1\end{array}$ & $\begin{array}{l}\overline{2} \\
1\end{array}$ & $\frac{1}{-}$ & $\frac{1}{-}$ & $\frac{1}{-}$ & $\begin{array}{l}8 \\
9 \\
3\end{array}$ \\
\hline Total & 5 & 9 & 3 & 1 & 1 & 1 & 20 \\
\hline
\end{tabular}

TABELA 9

Distribuição dos pacientes infartados, internados em UTI, segunđo o dia de internação e a d(A.D) da pressão arterial sistólica. São Paulo, 1976

\begin{tabular}{|c|c|c|c|c|c|c|c|c|}
\hline 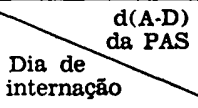 & $\begin{array}{r}\text { dife } \\
\text { pos } \\
0-\mid 10\end{array}$ & $\begin{array}{l}\text { nça } \\
\text { Iva } \\
10-\mid 20\end{array}$ & $0-10$ & $\begin{array}{l}\text { diferençe } \\
10-\mid 20\end{array}$ & $\begin{array}{l}\text { negativ } \\
20-130\end{array}$ & $30-140$ & $\begin{array}{c}\text { diferença } \\
\text { nula }\end{array}$ & Total \\
\hline $\begin{array}{l}1 .^{\circ} \\
2 .^{\circ} \\
3 .^{\circ}\end{array}$ & $\begin{array}{r}1 \\
2 \\
-\end{array}$ & $\frac{2}{-}$ & $\begin{array}{r}2 \\
2 \\
-\end{array}$ & $\begin{array}{r}1 \\
3 \\
-\end{array}$ & $\frac{1}{1}$ & $\frac{-}{1}$ & $\begin{array}{l}1 \\
2 \\
1\end{array}$ & $\begin{array}{l}8 \\
9 \\
3\end{array}$ \\
\hline Total & 3 & 2 & 4 & 4 & 2 & 1 & 4 & 20 \\
\hline
\end{tabular}

TABELA 10

Distribuição dos pacientes infartados, internados em UTI, segundo o dia de internaçăo e d(A-D) da pressåo arterial diastólica. São Paulo, 1976

\begin{tabular}{|c|c|c|c|c|c|c|}
\hline$\underbrace{d e}_{\text {Dia }} d_{d a}^{d(A-D)}$ PAD & $\begin{array}{r}d \\
0-110\end{array}$ & $\begin{array}{l}\text { ça } \\
\text { va } \\
10-\mid 20\end{array}$ & $\underset{0-10}{n}$ & $\begin{array}{l}\text { aça } \\
\text { va } \\
10-120\end{array}$ & $\begin{array}{c}\text { diferença } \\
\text { nula }\end{array}$ & Total \\
\hline $\begin{array}{l}1.0^{\circ} \\
2 .^{\circ} \\
3.0^{\circ}\end{array}$ & $\frac{1}{-}$ & $\frac{1}{-}$ & $\begin{array}{l}1 \\
4 \\
1\end{array}$ & $\begin{array}{l}3 \\
1 \\
1\end{array}$ & $\begin{array}{l}2 \\
4 \\
1\end{array}$ & $\begin{array}{l}8 \\
9 \\
3\end{array}$ \\
\hline Total & 1 & 1 & 6 & 5 & 7 & 20 \\
\hline
\end{tabular}

Nas tabelas 8, 9 e 10, observamos que as alterações foram mais acentuadas no primeiro dia de internação, sugerindo que a ansiedade é maior nos primeiros dias 
após a falência cardiaca aguda, o que vai de encontro ao achado de DELLIPIANI ${ }^{1}$, no qual o padrão de ansiedade em pacientes infartados encontrava-se elevado no início da doença, caindo nos primeiros dias.

TABELA 11

Distribuição dos pacientes infartados, internados em UTI, segundo o grau de parentesco e a d(A-D) da frequiência cardfaca. Săo Paulo, 1976

\begin{tabular}{|c|c|c|c|c|c|c|c|}
\hline $\begin{array}{l}\text { Grau de } \\
\text { parentesco }\end{array}$ & zero & $0-10$ & $10-\mid 20$ & $20-\mid 30$ & $30-\mid 40$ & $40-150$ & Total \\
\hline Conjuge & 3 & 5 & 3 & - & 1 & 1 & 13 \\
\hline Filho & 1 & 2 & - & 1 & - & - & 4 \\
\hline Irmão & 1 & 1 & - & - & - & - & 2 \\
\hline Neto & - & 1 & - & - & - & - & 1 \\
\hline Total & 5 & 9 & 3 & 1 & 1 & 1 & 20 \\
\hline
\end{tabular}

TABELA 12

Distribulção dos pacientes infartados, internados em UTI, segundo o grau de parentesco e a d(A-D) da pressão arterial sistólica. São Paulo, 1976

\begin{tabular}{|c|c|c|c|c|c|c|c|c|}
\hline$\underset{\text { prau de }}{\text { Grentesco }} d$ PAS & \multicolumn{2}{|c|}{$\begin{array}{l}\text { Diferença } \\
\text { positiva } \\
0-|10 \quad 10-| 20\end{array}$} & \multicolumn{4}{|c|}{ Diferença negativa } & $\begin{array}{l}\text { Diferença } \\
\text { nula }\end{array}$ & Total \\
\hline Cônjuge & 2 & 2 & 1 & 3 & 1 & 1 & 3 & 13 \\
\hline Fillho & - & - & 1 & 1 & 1 & - & 1 & 4 \\
\hline Irmäo & - & - & 2 & - & - & - & - & 2 \\
\hline Neto & 1 & - & - & - & - & - & - & 1 \\
\hline Total & 3 & 2 & 4 & 4 & 2 & 1 & 4 & 20 \\
\hline
\end{tabular}

TABELA 13

Distribuiçăo dos pacientes infartados, internados em UTI, segundo o grau de parentesco e a d(A-D) da pressâo arterial diastólica. São Paulo, 1976

\begin{tabular}{|c|c|c|c|c|c|c|}
\hline$\underset{\text { prarentesco }}{\text { Grau de }} d($ PAD $d a$ & \multicolumn{2}{|c|}{$\begin{array}{l}\text { Diferença } \\
\text { positiva }\end{array}$} & \multicolumn{2}{|c|}{$\begin{array}{l}\text { Diferença } \\
\text { negativa }\end{array}$} & $\begin{array}{l}\text { Diferença } \\
\text { nula }\end{array}$ & Total \\
\hline Cônjuge & 1 & 1 & 3 & 2 & 6 & 13 \\
\hline Filho & - & - & 1 & 2 & 1 & 4 \\
\hline Irmão & - & - & 1 & 1 & - & 2 \\
\hline Neto & - & - & 1 & - & - & 1 \\
\hline Total & 1 & 1 & 6 & $\mathbf{5}$ & 7 & 20 \\
\hline
\end{tabular}

Excetuando-se a PAD, os demais parâmetros tiveram sua alteração mais evidente nos pacientes que receberam visitas de cônjuge e filho, do que nos pacientes que receberam outra pessoa da família.

Verificamos ainda que dos pacientes que apresentaram crise de choro, quatro receberam visitas de esposa e dois de filhos, sugerindo que quanto maior a proximidade de parentesco, melhor se evidencia a reação emocional. 
TABELA 14

Distribuição dos pacientes infartados, internados em UTI, segundo o tempo de permanência do visitante e a $d(A \cdot D)$ da frequêencia cardíaca. São Paulo, 1976

\begin{tabular}{|c|c|c|c|c|c|c|c|}
\hline $\begin{array}{l}\text { Tempo de } \\
\text { permanência }\end{array}$ & zero & $0-10$ & $10-\mid 20$ & $20-30$ & $30-\mid 40$ & $40-i 50$ & Total \\
\hline $\begin{array}{l}0 \mathrm{~min} .30 \mathrm{~min} \\
30 \mathrm{~min} .60 \mathrm{~min} \\
60 \mathrm{~min}-90 \mathrm{~min} \\
90 \mathrm{~min}-120 \mathrm{~min}\end{array}$ & $\begin{array}{l}4 \\
1 \\
-\end{array}$ & $\frac{4}{1}$ & $\begin{array}{l}2 \\
1 \\
-\end{array}$ & $\begin{array}{l}1 \\
-\end{array}$ & $\frac{1}{-}$ & $\frac{1}{-}$ & $\begin{array}{c}13 \\
2 \\
1 \\
4\end{array}$ \\
\hline Total & 5 & 9 & 3 & 1 & 1 & 1 & 20 \\
\hline
\end{tabular}

TABELA 15

Distribuição dos pacientes infartados, internados em UTI, segundo o tempo de permanência do visitante e a d(A-D) da pressão arterial sistólica. São Paulo, 1976

\begin{tabular}{|c|c|c|c|c|c|c|c|c|}
\hline $\begin{array}{l}\text { Tempo de } \\
\text { permanência }\end{array}$ & \multicolumn{2}{|c|}{$\begin{array}{l}\text { Diferença } \\
\text { positiva } \\
0-10 \quad 10-\mid 20\end{array}$} & $0-\mid 10$ & \multicolumn{3}{|c|}{$\begin{array}{l}\text { Diferença negativa } \\
10-|20 \quad 20-| 30 \quad 30-\mid 40\end{array}$} & $\begin{array}{l}\text { Diferença } \\
\text { nula }\end{array}$ & Total \\
\hline $\begin{array}{l}0 \mathrm{~min} .30 \mathrm{~min} \\
30 \mathrm{~min}-60 \mathrm{~min} \\
60 \mathrm{~min} .90 \mathrm{~min} \\
90 \mathrm{~min}-120 \mathrm{~min}\end{array}$ & $\begin{array}{l}2 \\
1 \\
1\end{array}$ & $\frac{-}{1}$ & $\frac{4}{1}$ & $\begin{array}{l}3 \\
- \\
-\end{array}$ & $\begin{array}{l}2 \\
- \\
-\end{array}$ & $\bar{z}$ & $\begin{array}{l}2 \\
1 \\
1 \\
-\end{array}$ & $\begin{array}{r}13 \\
2 \\
1 \\
4\end{array}$ \\
\hline Total & 4 & 1 & 5 & 3 & 2 & 1 & 4 & 20 \\
\hline
\end{tabular}

TABELA 16

Distribuição dos pacientes infartados, internados em UTI, segundo o tempo de permanência do visitante e a d(A-D) da pressão arterial diastólica. São Paulo, 1976

\begin{tabular}{|c|c|c|c|c|c|c|}
\hline \multirow{2}{*}{$\begin{array}{l}\text { Tempo de } \\
\text { permanência }\end{array}$} & & & \multirow[t]{2}{*}{$\begin{array}{l}\text { Diferença } \\
\text { nula }\end{array}$} & \multirow[b]{2}{*}{ Tota } \\
\hline & $0-110^{5}$ & $10-20$ & $0-10$ & $10-20$ & & \\
\hline $\begin{array}{l}0 \mathrm{~min}-30 \mathrm{~min} \\
30 \mathrm{~min}-60 \mathrm{~min} \\
60 \mathrm{~min}-90 \mathrm{~min} \\
90 \mathrm{~min}-120 \mathrm{~min}\end{array}$ & $\frac{-}{1}$ & $\frac{-}{1}$ & $\frac{4}{-}$ & $\begin{array}{l}5 \\
- \\
-\end{array}$ & $\begin{array}{r}4 \\
2 \\
1 \\
-\end{array}$ & $\begin{array}{r}13 \\
2 \\
1 \\
4\end{array}$ \\
\hline Total & 1 & 1 & 6 & 5 & 7 & 20 \\
\hline
\end{tabular}

As tabelas 14, 15 e 16 mostram que o tempo de permanência do visitante variou de um minimo de 5 minutos a um máximo de 105 minutos; observamos, ainda, que um número maior de visitantes (13) permaneceu durante o período de 0 a 30 minutos, ocorrendo neste periodo as mudanças mais acentuadas da FC, PAS e PAD. Este fato nos levou a supor que a alteração possa ocorrer no impacto inicial do contacto paciente-visitante.

\section{CONCLUSÕES}

O presente estudo permitiu concluir que os pacientes reagem emocionalmente à visita, uma vez que houve mudanças significativas na freqüência cardiaca e na pressão arterial sistólica e diastólica após a visita de familiares.

Considerando que este estudo foi feito com uma amostra pequena, julgamos ser precipitado generalizar as conclusões obtidas. Entretanto, no nosso estudo houve 
relação entre a idade e o dia de internação do paciente com a visita de familiares e as alterações de seus parâmetros cardiovasculares. 0 grau de parentesco, possivelmente, deve contribuir também para a ocorrência de tais alterações.

Outrossim, há indícios sugestivos de que quanto menor o tempo de permanência do visitante junto ao paciente, maiores são as alterações na $\mathbf{F C}$ e $\mathbf{P A}$, Isto nos leva a supor que no impacto inicial do contacto paciente-visitante, poderia ocorrer uma alteração nos parâmetros cardiovasculares e, a medida que a visita fosse transcorrendo, esses valores retornariam aos níveis anteriores.

Essa última observação, aliada à necessidade de aumentar a amostra, nos conduziu a um novo estudo, com o qual pretendemos a obtenção de dados mais válidos.

TAKAHASHI, E. I. U. Cardiovasculares parameters' observation in infarted patients face relative's visit. Rev. Esc. Enf. USP, São Paulo, 14(1):9-19, 1980.

This survey was made with 20 infarcted patients; and their blood pressure and cardiac rate were examined before and after the visit of relative. It was verified that they showed alterations due to emotional situations.

\section{REFERENCIAS BIBLIOGRAFICAS}

1. DELLIPIANI, A. V. et alii. Anxiety after a heart attack. British Heart J., London, 38: 752-7, 1976,

2. LEE, R. E. \& BALL, P. A. Some thoughts on the psychology of the coronary care unit patient. American Journal of Nursing, New York 75(9): 1498-1501, 1975.

3. SECOR, J. Coronary care - a nursing specialty. New York, Meredith Corporation, 1971. 
ANEXO 1

Ficha para coleta de dados Ficha n.o:

Data:

\begin{tabular}{|c|c|c|c|c|}
\hline $\begin{array}{l}\text { I. IDENTIFICAGaO } \\
\text { Nome: } \\
\text { Data de internação: }\end{array}$ & Sexo: & Idade: & $\begin{array}{l}\text { Cor: } \\
\text { Dia de internaçăa: }\end{array}$ & Estado civil: \\
\hline $\begin{array}{l}\text { II. CONTROLES } \\
\text { 1." Mensuragåo ("antes") }\end{array}$ & $\mid \begin{array}{l}\mathbf{P A} \\
\mathbf{F C}\end{array}$ & $\mid \begin{array}{l}1 . * \\
2 . *\end{array}$ & & \\
\hline 2." Mensuraçäo ("depois") & $\mid \begin{array}{l}\mathbf{P A} \\
\mathbf{F C}\end{array}$ & $\left|\begin{array}{l}1.9 \\
2.2\end{array}\right|$ & & \\
\hline $\begin{array}{l}\text { III. VISITAS } \\
\text { Visitantes: } \\
\text { Hora de chegada: } \\
\text { Tempo de permanencia: }\end{array}$ & & & Hora de safda & \\
\hline
\end{tabular}

TV. OBSERVACOES 


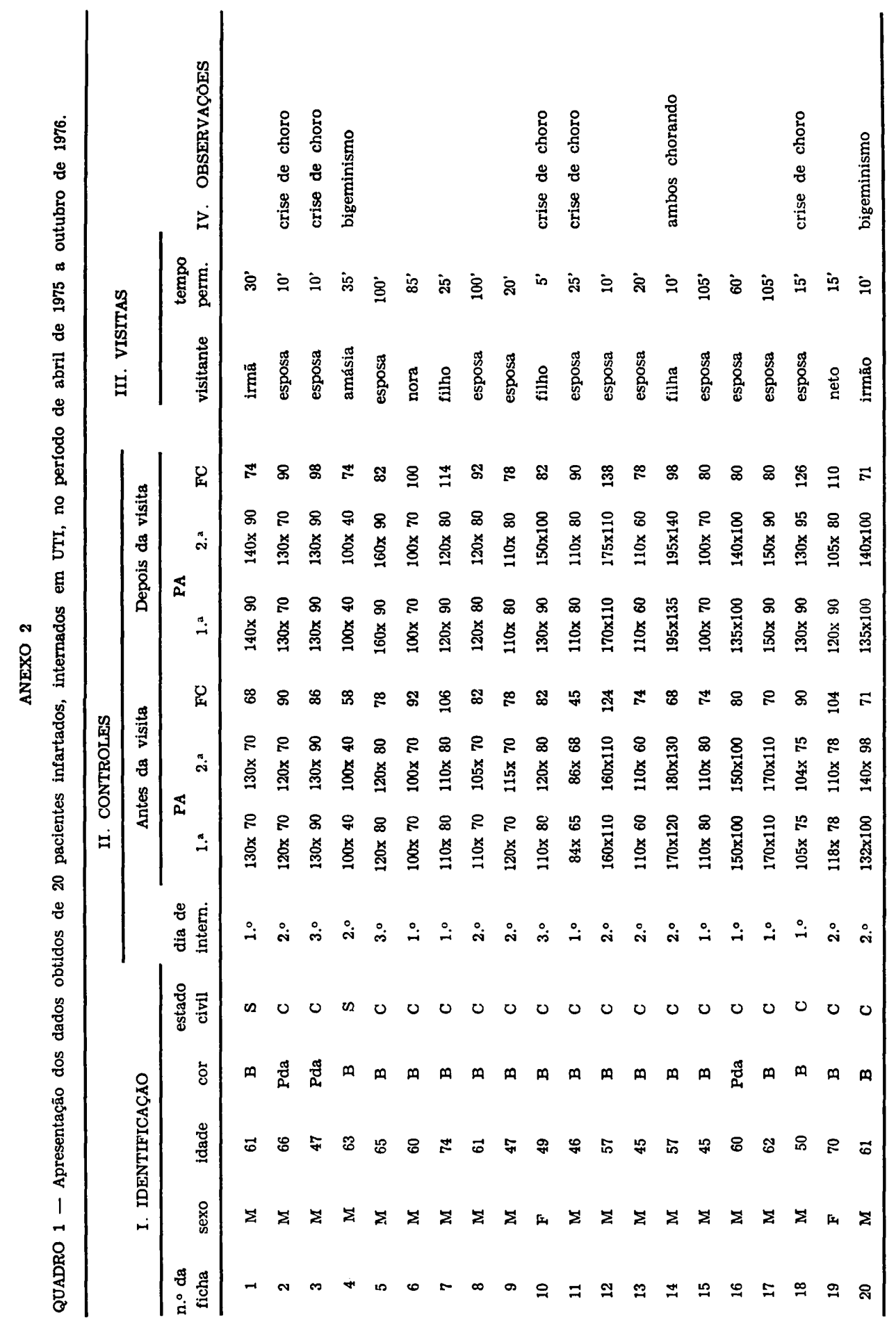

